\section{Iris configuration in accommodation in pigment dispersion syndrome}

MO Balidis, ${ }^{1,2}$ C Bunce, ${ }^{1}$ CJ Sandy, ${ }^{1}$ RPL Wormald ${ }^{1}$ and $\mathrm{MH}$ Miller ${ }^{1}$
${ }^{1}$ Moorfields Eye Hospital

London, UK

${ }^{2}$ Department of

Ophthalmology

University of Thessaloniki

Medical School

Thessaloniki, Greece

Correspondence:

MO Balidis

Ermou 46

54623 Thessaloniki

Greece

E-mail: balidis@otenet.gr

The work has been presented at The

Association for Research in Vision and Ophthalmology (ARVO) Annual Meeting,

Fort Lauderdale, Florida, May 1999

Received: 7 August 2001 Accepted: 16 January 2002

\begin{abstract}
Purpose This study was conducted in order to investigate the effect of accommodation on the iris morphology and iridolenticular contact, in eyes with Pigment Dispersion Syndrome and Pigmentary Glaucoma, using high-resolution ultrasound.

Methods We performed a prospective observational study, examining a group of 30 Pigment Dispersion Syndrome and Pigmentary Glaucoma patients (49 eyes) and a smaller group of eight 'normals' nonPigment Dispersion Syndrome patients (8 eyes). All patients underwent ultrasound biomicroscopy, before and during accommodation.

Results The iris profile before accommodation was found convex in $48.5 \%$, flat in $\mathbf{1 9 . 7 \%}$ and concave in $\mathbf{3 1 . 8 \%}$.

Following accommodation the iris configuration remained unchanged in $66.2 \%$, increased in concavity in $20.3 \%$ and decreased in concavity in $\mathbf{1 3 . 5 \%}$. Conclusion The effect of accommodation on iris configuration and accommodation is highly variable. From our experience the measurement of iris configuration using ultrasound biomicroscopy may not be a useful method of evaluating the effect of different treatments on iris configuration. Eye (2002) 16, 694-700. doi:10.1038/ sj.eye. 6700115
\end{abstract}

Keywords: accommodation; pigmentary glaucoma; pigment dispersion syndrome; iris configuration; ultrasound biomicroscopy; highresolution ultrasound

\section{Introduction}

Pigment Dispersion Syndrome is a condition characterised by loss of pigment from the neuroepithelial posterior surface of the iris.
The free pigment particles spread throughout various structures of both anterior and posterior chambers including the zonules, the anterior and posterior surfaces of the lens, the iris, the cornea and more importantly the trabecular meshwork. The classic diagnostic triad for pigment dispersion syndrome (PDS) is:

(1) Iris chafing with typical midperipheral spoke-like transillumination defects;

(2) Deposition of pigment on the corneal endothelium, in spindle-shape pattern (Kruckenberg spindle);

(3) Dense pigmented band of trabecular meshwork (which gonioscopically appears homogenous, at least at the active stages), causing decreased outflow capacity of the meshwork.

In 1979, Campbell ${ }^{1}$ proposed a pathophysiological process resulting in the release of pigment. His theory was based on an observation of posterior bowing of the iris in eyes with PDS, so enabling the pigment epithelium to contact the zonules. Campbell postulated that reversal of this concavity would be the key to the management of PDS and suggested the use of peripheral iridotomies to relieve posterior bowing, mainly by equalising the pressure between anterior and posterior chambers. Biometric measurements of central and peripheral anterior chambers in patients with $\mathrm{PDS}^{2-4}$ support this theory. Ultrasound biomicroscopy imaging has received much interest from those researching anterior and middle ocular segments of the eye. Iris concavity has been shown in a proportion of patients with PDS. ${ }^{5}$ In Pavlin et al's study, high-resolution ultrasonography was used to evaluate the efficacy of peripheral iridotomy or topical pilocarpine on the profile of the iris.

Our study was conducted in order to examine iris configuration and iridolenticular 
contact using a new measurement technique and to assess whether either is influenced by accommodation.

\section{Methods}

We examined a group with Pigment Dispersion Syndrome (30 patients, 49 eyes) and a smaller group of eight controls non-Pigment Dispersion Syndrome (8 subjects, 8 eyes). In the PDS group 13 patients were on no treatment, 14 on one topical antiglaucoma medication, one on two medications (B-blocker, pilocarpine), one had received laser trabeculoplasty treatment and one had undergone a surgical filtering procedure. In our analysis we excluded the patient who underwent trabeculectomy and have highlighted in the tables and plots, the four individuals on miotic therapy (pilocarpine).

All subjects were recruited from the Glaucoma clinic at Moorfields Eye Hospital, London, UK. Examinations were conducted using the commercial version of Ultrasound Biomicroscopy (Humphrey Inc UBM, San Leandro, CA, USA) using a $50 \mathrm{MHz}$ frequency transducer, with the patient in supine position. High resolution ultrasound is capable of achieving lateral resolution of approximately $40 \mu \mathrm{m}$ with tissue penetration of about $4-5 \mathrm{~mm}$, producing images of $5 \times$ $5 \mathrm{~mm}$ field. We scanned each eye in four different meridians in a radial fashion at the limbus at approximately 90, 180, 270 and 360 degrees. At the first scanning examination, patients were asked to fixate with their fellow eye on a target 2 metres away (accommodation relaxed). During the second examination (accommodation induced), the patient's fellow eye fixated at a print target at $30 \mathrm{~cm}$ distance. During both examinations the non-examined eye had the full refractive correction (either wearing contact lenses or with a trial lens, with the appropriate adjustments of back vertex distance). We first scanned the meridians at 3 o'clock, then continued clockwise at 6, 9 and 12 o'clock. We repeated the same procedure, reintroducing accommodation in each scan, in each meridian, in order to avoid any variation in accommodation. Room lighting was held constant to ensure that pupil size remained unchanged by the light reflex, in the accommodating eye. The patients had an interval of 30-45 min between each examination where they were allowed to read. Before the examination there was a period of 5-10 min for preparation of the patient (examination in flat position, application of topical anaesthesia, insertion and filling of eye cup) where the fellow eye was not in any accommodative state. As explained by Pavlin's theory of loss of posterior bowing after prolonged accommodation, with increasing time the eye is entering a stable state, with an equalised pressure between AC and PC. The stable state eye was then stimulated to accommodate and induce posterior bowing.

We devised a new technique for measuring iris configuration that makes use of readily identifiable anatomical features of the eye. Figure 1 illustrates this method. The outermost point of iridolenticular contact: $A$, is joined by a straight line: $A B$, to the peripheral end of the iris pigment epithelium IPE: $B$. The point of

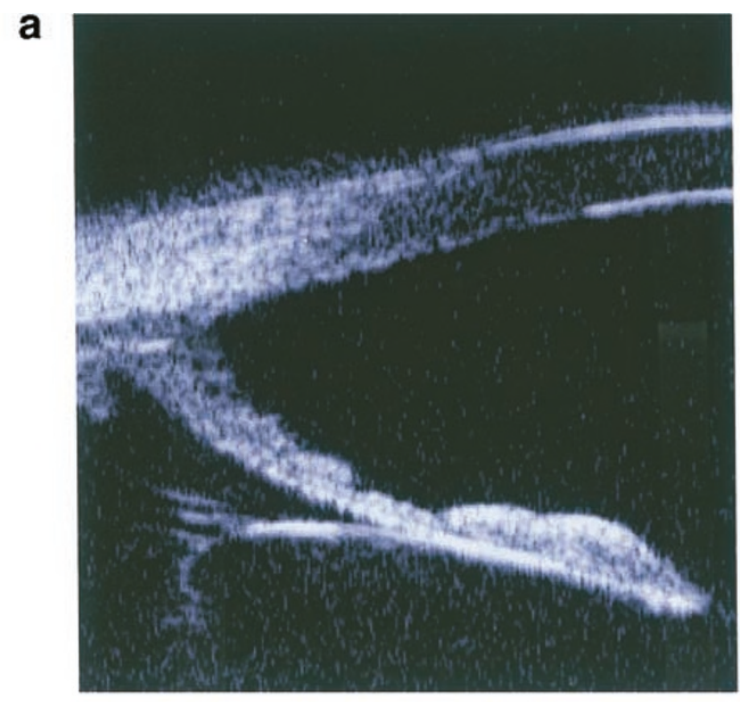

b

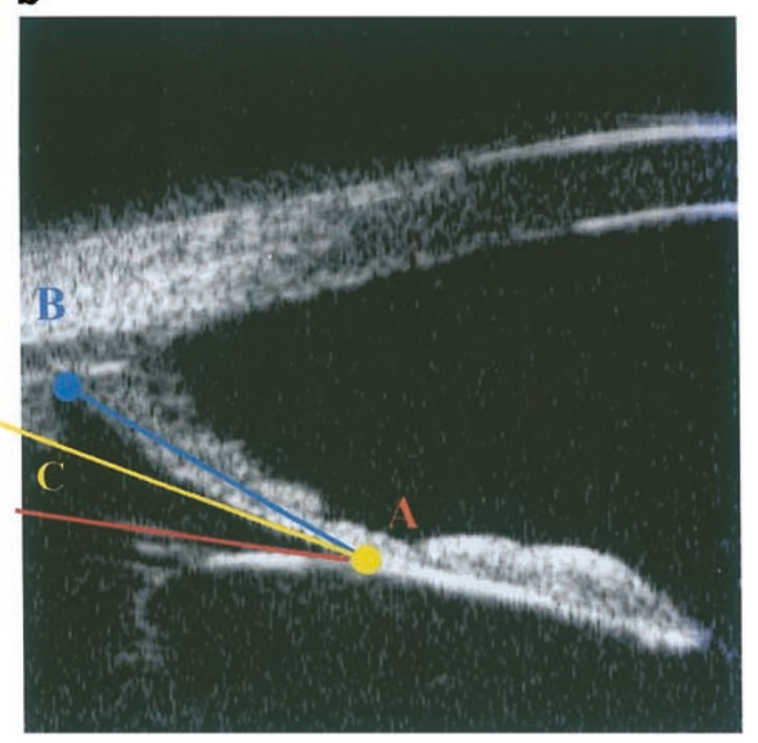

Figure 1 R/D score-method of measuring iris configuration. (a) Posterior bowing configuration in a PDS eye. (b) Same scan as in (a) with application of R/D score method of measurement. The outermost point of iridolenticular contact: $A$, is joined by a straight line: $A B$, to the peripheral end of the iris pigment epithelium IPE: $B$. The point of greatest displacement of the iris $C$ (usually found at the midperiphery), is joined to $A$ by the straight line $A C$. The third line is the tangent line of the lens at point $A$. The two angles created are then measured. 
greatest displacement of the iris (either posterior or anterior): $C$ (usually found at the midperiphery), is joined to $A$ by the straight line $A C$. Two angles are then measured. The first, termed the reference angle, $R$, is the angle between the tangent of the lens at point $A$ to line $A B$. The second, termed the displacement angle, $D$, is the angle between the tangent of the lens at point $A$ to the line $A C$. In an eye with a planar configuration, the ratio $R / D$ will equal 1 . The greater the ratio, the greater the concavity of the iris.

The reproducibility of the applied method was evaluated by inter- and intra-observer assessment of the R/D scores. Intra-observer variability was small; the average differences between first and second scores of each observer being less than 0.01 units. Percentage agreement within observers was $89 \%$ or higher, with kappa values of 0.8 or higher, indicating almost perfect agreement. Inter-observer variability was greater but there was substantial agreement between the three observers.

\section{Statistical methods}

Descriptive techniques only were employed. All analyses were conducted using STATA. ${ }^{6}$ Scatter diagrams were plotted to display actual R/D scores. To be consistent with other papers, we categorised $\mathrm{R} / \mathrm{D}$ scores as:

(i) Anterior Bowing (convex) if the R/D score was $\leqslant 0.9$;

(ii) Flat if the R/D score was $>0.9$ and $<1.1$;

(iii) Posterior Bowing (concave) if the $\mathrm{R} / \mathrm{D}$ score was $\geqslant 1.1$.

Table 1 presents the demographics of the PDS population under study. Figures $2 \mathrm{a}, \mathrm{b}$ and $3 \mathrm{a}, \mathrm{b}$ are

1360 meridian

- 180 meridian

HORIZONTAL

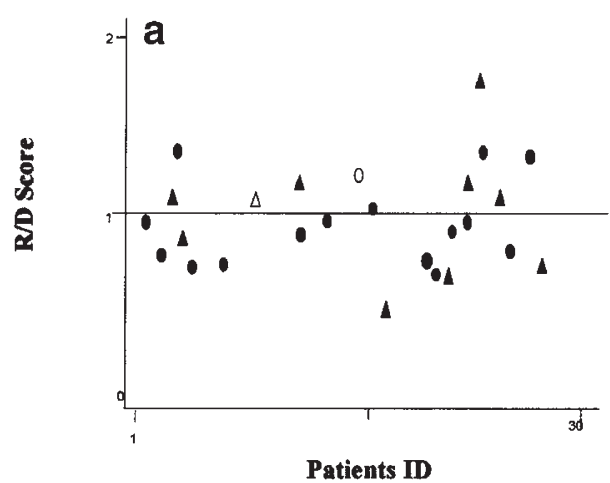

scatter plots of R/D scores for iris configuration and iridolenticular contact (ILC) recorded on the PDS eyes before accommodation, at horizontal (Figures 2a, 3a) and vertical (Figure 2b, 3b) meridians. It should be noted that in many cases less than the study numbers have been plotted. This was because not all angle scans could be assessed-therefore we recommend more than one scan per eye, to ensure measurable results. Table 2 tabulates the concavity of eyes pre- and postaccommodation in each angle. Table 3 summarises change in iris configuration after accommodation. Figure $4 \mathrm{a}$ and $\mathrm{b}$ are scatter plots of the change in iris profile after accommodation ( $\mathrm{R} / \mathrm{D}$ score during minus $\mathrm{R} / \mathrm{D}$ score before) against age. Positive values represent increased concavity and negative the reverse. Figures $5 \mathrm{a}$ and $\mathrm{b}$ present R/D scores in the 90 degree and 180 degree images of 'normal' eyes before and during accommodation.

\section{Results}

Table 1 shows that the majority $(70 \%)$ of the PDS patients were male and that the average age of the patients was 53 years. The oldest patient in the study was 75 years and the youngest 31 years. The difference in age between men and women was not statistically significant (men were on average slightly older than the women). More left eyes were examined than right eyes. This was because ten patients ( 8 men) refused to have their second eye scanned-and in only one of those was the second eye to be tested the left eye. Average IOP levels were similar between men and women, the median being $18 \mathrm{mmHg}$ (IQR: 16, 20). Most patients were moderately myopic (Table 1 ).

Table 2 summarises the distribution of iris configurations, with and without accommodation, in

$\triangle 270$ meridian

- 90 meridian
VERTICAL

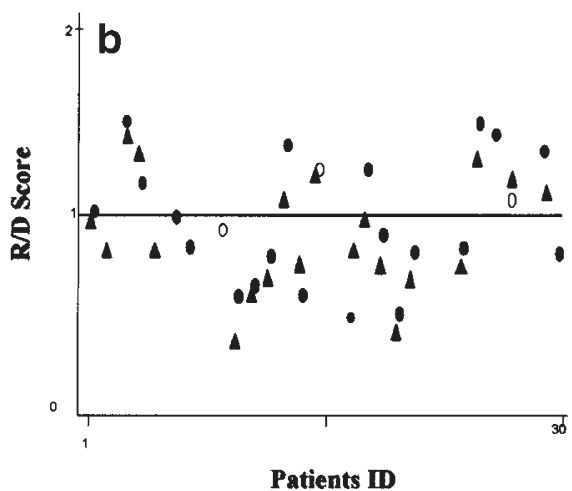

Figure 2 Iris configuration in PDS patients. (a, b) Scattered plots of iris configuration in patients with Pigment Dispersion Syndrome. Triangles and circles not filled represent patients on pilocarpine treatment. 

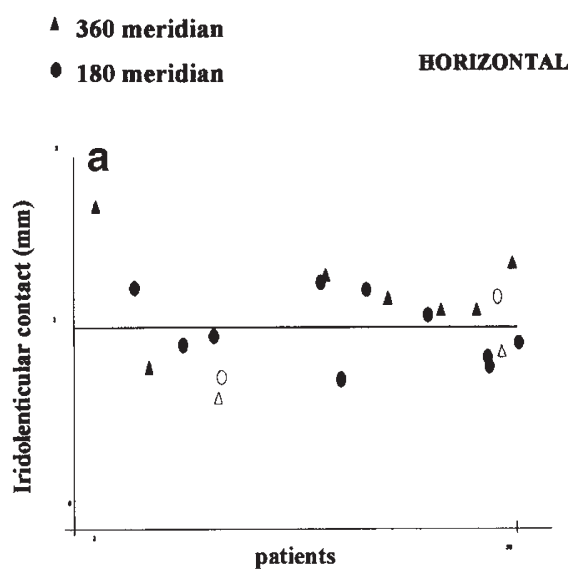

490 meridian

- 270 meridian

VERTICAL

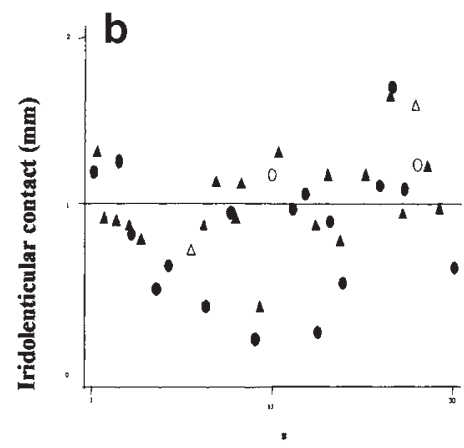

Figure 3 Iridolenticular contact (ILC) in PDS patients. (a, b) Scattered plots of ILC in patients with Pigment Dispersion Syndrome. Triangles and circles not filled represent patients in pilocarpine treatment.
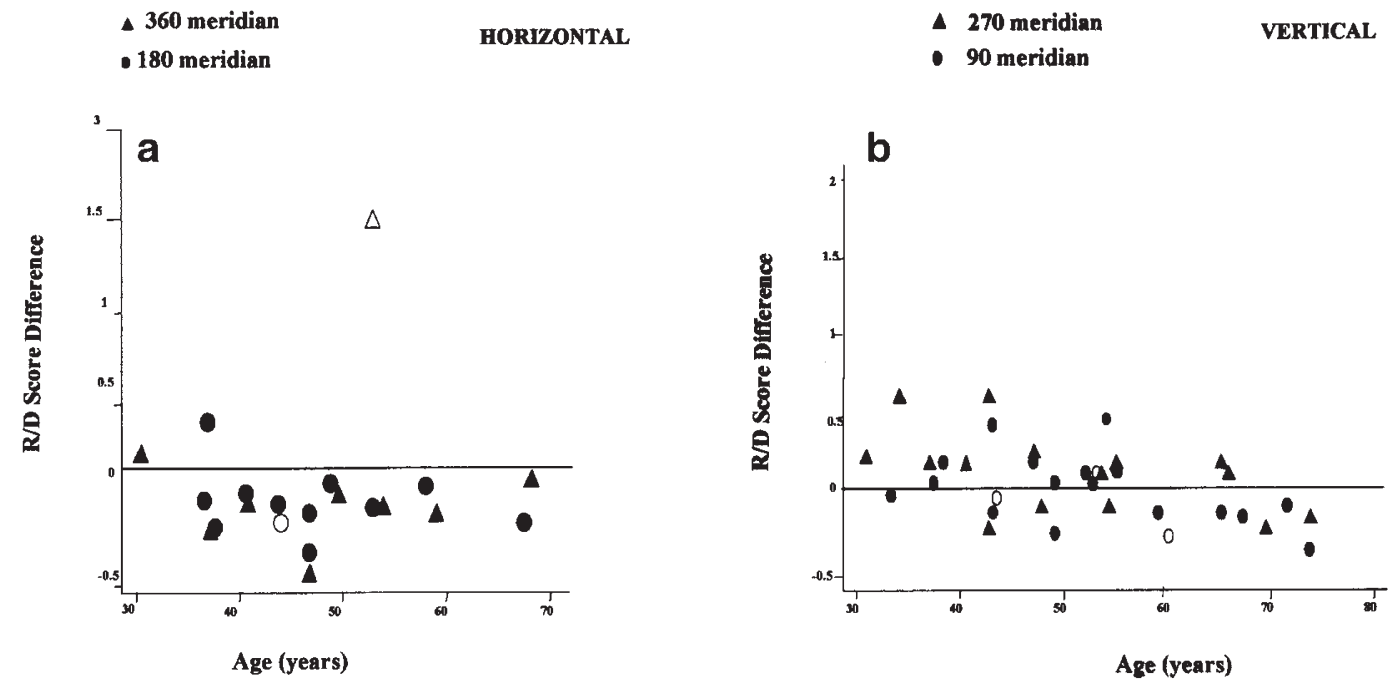

Figure 4 Change in iris configuration induced by accommodation against age in PDS eyes. (a, b) Scattered plots of changes in iris configuration induced by accommodation against age in PDS eyes. Triangles and circles not filled represent patients on pilocarpine treatment.

each of the four meridians. Table 3 tabulates iris profile changes, induced by approximately 3.3 diopters of accommodation (in most of our patients), in the same quadrant of the same eye. In the majority (66.7\%) of the scanned sections, iris configuration remained unchanged. Posterior bowing increased in $31.25 \%$ of the eyes, at 270 degrees (6 o'clock).

Subsequent analyses were conducted using one eye from each patient. Where both eyes of a patient had been scanned, we randomly selected equal numbers of right and left eyes. Figures $2 \mathrm{a}, \mathrm{b}$ and $3 \mathrm{a}, \mathrm{b}$ clearly show that there is a range of concavity in these eyes and even scanning the same eyes, different meridians show different scores.

Figures $4 \mathrm{a}$ and $\mathrm{b}$ suggest that the change in iris configuration during accommodation in eyes with PDS is quite variable. Whilst iris concavity increases in some, it decreases or remains unchanged in others. Despite controlling for accommodation, many of the images showed no evidence of posterior bowing. These figures demonstrate some concavity in the iris in eyes of patients aged 70 or more, so that age seems unlikely to be the sole determinant of such variability.

Figures $5 \mathrm{a}$ and $\mathrm{b}$ illustrate that iris concavity is also present in non-PDS eyes without accommodation. From the scatter plot is apparent that whilst accommodation increases the concavity in some subjects, the reverse is seen in others.

\section{Discussion}

The linking of iridozonular rubbing to iris concavity in pigment dispersion syndrome led to the increasing use 
- with accommodation

$\Delta$ without accommodation

HORIZONTAL

a

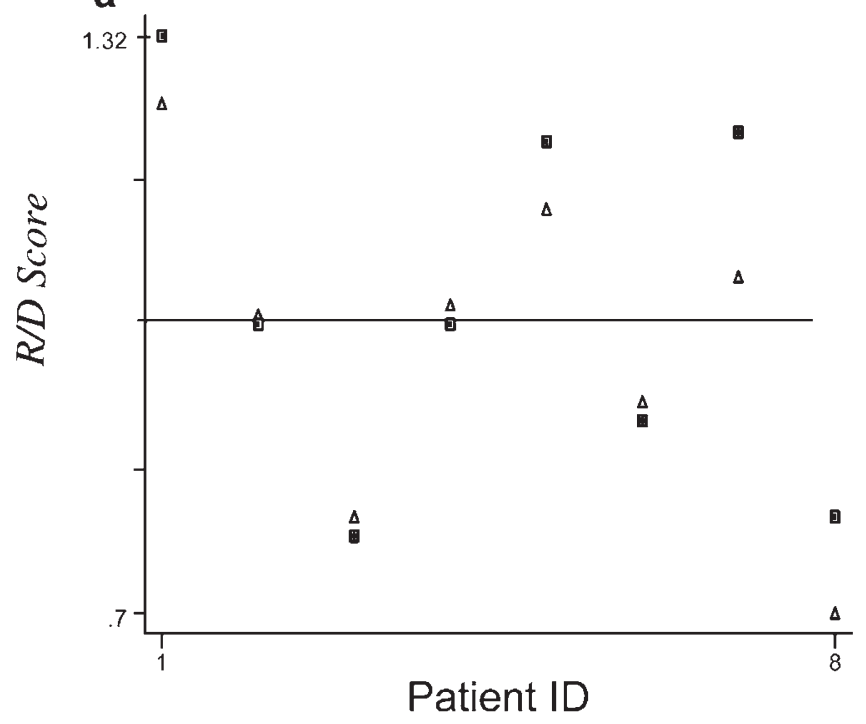

- with accommodation without accommodation

b

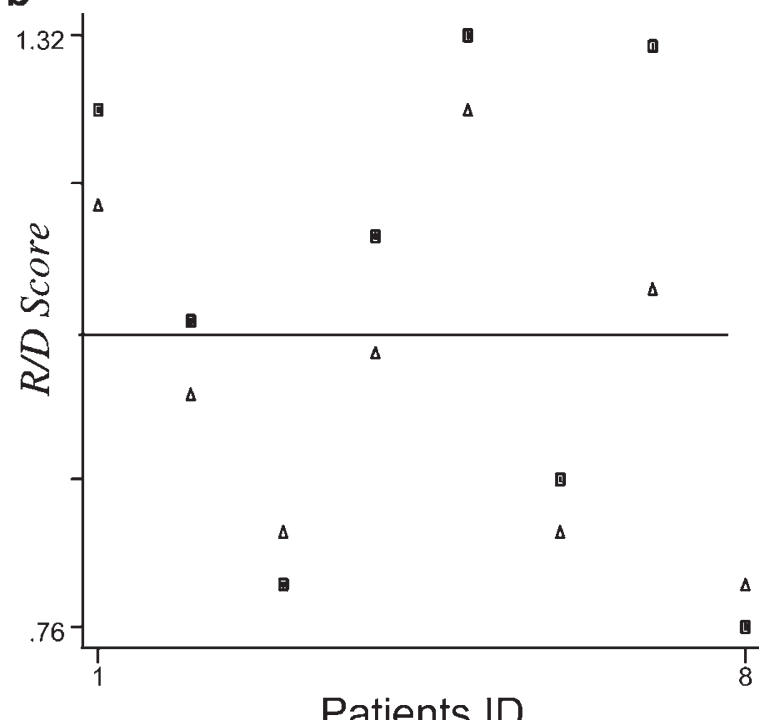

Figure 5 Iris configuration before and after accommodation in 'non PDS'. Iris concavity is present in some of the non-PDS eyes before accommodation and whilst accommodation increases the concavity in some subjects, the reverse is seen in others.

Table 1 Demographics of the studied population

\begin{tabular}{llll}
\hline Sex: No. (\%) & $\begin{array}{l}\text { Male } 21 \\
(70 \%)\end{array}$ & $\begin{array}{l}\text { Female } 9 \\
(30 \%)\end{array}$ & $\begin{array}{l}\text { Total } \\
30\end{array}$ \\
\hline Age (years): median (IQR) & $54(44,66)$ & $48(43,53)$ & $53(44,59)$ \\
Eyes & $14 \mathrm{RE}, 20 \mathrm{LE}$ & $7 \mathrm{RE}, 9 \mathrm{LE}$ & $21 \mathrm{RE}, 29 \mathrm{LE}$ \\
IOP (mmHg): median (IQR) & $18(17,20)$ & $17(15.5,19)$ & $18(16,20)$ \\
Ref: median (IQR) & $-3.13(-7.25,-0.75)$ & $-4.5(-6.25,-2.45)$ & $-3.75(-6.25,-1.5)$ \\
\hline
\end{tabular}

IOP, intraocular pressure (in $\mathrm{mmHg}$ ). Ref, refractive error. IQR, interquartile range.

Table 2 Iris concavity of eyes pre- and post-accommodation

\begin{tabular}{llll}
\hline Meridian & & \multicolumn{1}{c}{$\begin{array}{c}\text { Pre- } \\
\text { accommodation }\end{array}$} & $\begin{array}{c}\text { Post- } \\
\text { accommodation }\end{array}$ \\
\hline 90 & Anterior bow & $11(45.8 \%)$ & $8(34.8 \%)$ \\
& Flat & $4(16.7 \%)$ & $6(26.1 \%)$ \\
& Posterior bow & $9(37.5 \%)$ & $9(39.1 \%)$ \\
& Total eyes & 24 & 23 \\
180 & Anterior bow & $6(37.5 \%)$ & $8(47.1 \%)$ \\
& Flat & $5(31.25 \%)$ & $3(17.6 \%)$ \\
& Posterior bow & $5(31.25 \%)$ & $6(35.3 \%)$ \\
& Total eyes & 23 & 17 \\
& Anterior bow & $11(57.9 \%)$ & $7(41.2 \%)$ \\
& Flat & $3(15.8 \%)$ & $6(35.3 \%)$ \\
& Posterior bow & $5(26.3 \%)$ & $4(23.5 \%)$ \\
360 & Total eyes & 19 & 17 \\
& Anterior bow & $4(57.1 \%)$ & $5(50.0 \%)$ \\
& Flat & $1(14.3 \%)$ & $1(10.0 \%)$ \\
& Posterior bow & $2(28.6 \%)$ & $4(40.0 \%)$ \\
& Total eyes & 7 & 10 \\
\hline
\end{tabular}

Table 3 Change of iris configuration after accommodation in four meridians

\begin{tabular}{lcccc}
\hline & $\begin{array}{c}90 \\
n(\%)\end{array}$ & $\begin{array}{c}180 \\
n(\%)\end{array}$ & $\begin{array}{c}270 \\
n(\%)\end{array}$ & $\begin{array}{c}360 \\
n(\%)\end{array}$ \\
\hline $\begin{array}{l}\text { Increased } \\
\text { posterior } \\
\text { bowing }\end{array}$ & $4(18.2 \%)$ & $2(13.3 \%)$ & $5(31.25 \%)$ & $1(16.7 \%)$ \\
$\begin{array}{l}\text { Decreased } \\
\text { posterior } \\
\text { bowing }\end{array}$ & $4(18.2 \%)$ & $3(20.0 \%)$ & $1(6.25 \%)$ & $0(0.00 \%)$ \\
$\begin{array}{l}\mathbf{1}^{\mathrm{a}} \\
\text { Remained } \\
\text { unchanged }\end{array}$ & $14(63.6 \%)$ & $10(66.7 \%)$ & $10(62.5 \%)$ & $5(83.3 \%)$ \\
& $\mathbf{1}^{\mathrm{a}}$ & & & \\
$\begin{array}{l}\text { Total No. } \\
\text { of eyes }\end{array}$ & 22 & 15 & 16 & 6 \\
\hline
\end{tabular}

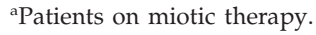


of prophylactic laser peripheral iridotomy. ${ }^{1,7}$ Since Campbell's original hypothesis, ${ }^{1,2}$ several studies have captured the posterior bowing of the iris using high resolution ultrasound in patients with pigment dispersion syndrome, and its reversal after laser (peripheral iridotomy) or medical (topical miotics) treatment. ${ }^{5,7,8}$

Our study raises doubt as to the validity of a single image of the eye. Images at different meridians, using the same settings of illumination and focusing, measured and scored by the same observer, taken sequentially, yield different R/D scores. Posterior bowing appears to be a dynamic phenomenon and as such its reproducibility in imaging can be a challenge. The state of the eye prior to the examination is affecting iris profile. Different factors can alter the final result. Pupillary diameter is one to consider during induction of posterior bowing. The valve effect can occur in a small pupil, but not in a dilated one. Also the use of eyecup, during scanning can have a flattening effect, possibly by preventing blinking.

From our experience accommodation is not the ideal method of inducing posterior bowing, or thereafter observing the effect of different treatment modalities in changing iris morphology. We induced approximately 3.3 diopters of accommodation during the second examination, in non-presbyopic eyes (fixation target at $30 \mathrm{~cm}$ distance from the fellow eye) and our rationale is that eyes do not accommodate greater than this in average daily activities. Thus if posterior bowing is responsible for the shedding of the iris pigment epithelium, it is clinically important to know whether we can demonstrate bowing in these eyes at this power of induced accommodation.

The presence of iris concavity in non-PDS patients has previously been attributed to change incurred by accommodation. Our study, albeit on a relatively lesser number of eyes, demonstrated iris concavity without accommodation. We observed concave irides in some meridians and convex anteriorly in others, when the eye was in the accommodative state, during the initial examination. Our study suggests that the association between iris configuration and accommodation is highly variable, some irides increase in concavity and others remain unchanged or decrease.

Numerous theories have appeared in the literature regarding the pathogenesis of PDS. We can hypothesise the existence of different anatomical subgroups within the syndrome. Posterior bowing may be a prominent anatomical feature in some but not all subgroups. Conditions that generally enlarge the globe have been reported to be associated with PDS or PG (Campbell, unpublished) and have coexisted in patients with megalocornea. ${ }^{9,10}$ A congenitally more posterior insertion of the iris in the ciliary body has been documented in PDS patients. ${ }^{11}$ Deeper anterior chamber, $^{3}$ mostly peripherally ${ }^{11}$ indicating a more generalised anatomical variation of the anterior third of the eye in Pigment Dispersion, may explain the unusually large posterior to anterior movement of the lens observed. Ocular pathologists ${ }^{12,13}$ detect displastic (and hyperplastic) changes in the anterior pigment epithelium of the iris, probably occurring as a primary congenital abnormality. The incidence of retinal peripheral degenerations ${ }^{14}$ (lattice) in patients with Pigment Dispersion Syndrome is higher, in all degrees of myopia, compared to the general population, and is also associated with a higher incidence of retinal detachment. Perhaps this congenitally transformed displastic epithelium of the middle segment of these eyes with PDS may be more vulnerable to rupture when in contact with the anterior zonules, resulting in release of pigmented granules.

Specialists in that field stated this sudden, dynamic backward bowing to be a transitory phenomenon, not easily identifiable in every case. Further work may reveal anatomical variations of the zonulo-capsular system (ie a more anterior insertion of zonules, possibly linking pigment dispersion with pseudoexfoliation syndrome (overlapping syndromes). Imaging of zonules requires higher resolution than available at present, in order to acquire accurate and reproducible measurements.

Our observations may dispute the theory that posterior bowing is the sole mechanism for pigment shedding. This paper stresses the significance of a multifactorial model of understanding pigment dispersion, where a single mechanism is incapable of explaining various points currently being discussed. In this hypothesis structural changes in the anterior third of the eye may be one of the phenotypic expressions of the 'PDS' gene. Up to now genetic researchers have identified, in one Irish family, a possible locus on the long arm of chromosome $7 .{ }^{15}$ Genetic mapping may reveal more than one location in human chromosomes, responsible for PDS in individuals from other ethnic backgrounds, and as a consequence more than one gene, responsible for the diversity of the syndrome's phenotype.

Experience from this study suggests that R/D scoring, by using prominent features of the UBM images, reduces the potential for inter- and intraobserver variability when measuring iris configuration. ${ }^{16,17}$ Further work is underway to assess this. 


\section{References}

1 Campbell Dg. Pigmentary dispersion and glaucoma. A new theory. Arch Ophthalmol 1979; 97: 1667-1672.

2 Campbell DG, Schertzer R. Pathophysiology of pigment dispersion syndrome and pigmentary glaucoma. Editorial review. Curr Op Ophthalmol 1995; 6: 96-101.

3 Davidson JA, Brubaker RF, Ilstrup DM. Dimensions of the anterior chamber in pigment dispersion syndrome. Arch Ophthalmol 1983; 101: 81-83.

4 Caprioli J, Spaeth GL, Wilson RP. Anterior chamber depth in open angle glaucoma. Br J Ophthalmol 1986; 70: 831-836.

5 Pavlin CJ, Macken P, Trope GE, Harasiewicz K, Foster FS. Accommodation and iridotomy in the pigment dispersion syndrome. Ophthalmic Surg Lasers 1996; 27: 113-120.

6 Stata Corporation. Stata Statistical Software: Release 5.0, 1997, College Station, TX, USA.

7 Karickhoff JR. Pigmentary dispersion syndrome and pigmentary glaucoma: a new mechanism concept, a new treatment, and a new technique. Ophthalmic Surg 1992; 23: 269-277.

8 Ritch R. A unification hypothesis of pigment dispersion syndrome. Trans Am Ophthalmol Soc 1996; 94: 381-389.

9 Cameron W. Kruckenberg spindle associated with megalocornea and posterior pigmentation of the lens. Am J Ophthalmol 1941; 24: 687-689.

10 Cavka V. Pigmentary glaucoma. Am J Ophthalmol 1961; 52: 880-894.

11 Sokol J, Stegman Z, Liebmann JM, Ritch R. Location of the iris insertion in pigment dispersion syndrome. Ophthalmology 1996; 103: 289-293.

12 Fine BS, Yanoff M, Scheie HG. Pigmentary glaucoma. A histologic study. Trans Am Acad Ophthalmol Otolaryngol 1974; 78: OP314-OP325.

13 Kupfer C, Kuwabara T, Kaiser-Kupfer M. The histopathology of pigmentary dispersion syndrome with glaucoma. Am J Ophthalmol 1975; 80: 857-862.

14 Scuderi G, Papale A, Nucci C, Cerulli L. Retinal involvement in pigment dispersion syndrome. Int Ophthalmol 1995; 19: 375-378.

15 Andersen $\mathrm{J}$ et al. A gene responsible for the pigment dispersion syndrome maps to chromosome 7q35-q36. Arch Ophthalmol 1997; 115: 384-388.

16 Spaeth GL, Azuara-Blanco A, Araujo SV, Augsburger JJ. Intraobserver and interobserver agreement in evaluating the anterior chamber angle configuration by ultrasound biomicroscopy. J Glaucoma 1997; 6; 13-17.

17 Tello C, Liebmann J, Potash SD, Cohen H, Ritch R. Measurement of ultrasound biomicroscopy images: intraobserver and interobserver reliability. Invest Ophthalmol Vis Sci 1994; 35: 3549-3552. 